\title{
Protocols for Thermoluminescence and Optically Stimulated Luminescence Research at DOSAR
}

October 2004

Prepared by

S. M. Bernal

University of Michigan

J. S. Bogard

Oak Ridge National Laboratory 


\title{
DOCUMENT AVAILABILITY
}

Reports produced after January 1, 1996, are generally available free via the U.S. Department of Energy (DOE) Information Bridge:

Web site: http://www.osti.gov/bridge

Reports produced before January 1, 1996, may be purchased by members of the public from the following source:

\author{
National Technical Information Service \\ 5285 Port Royal Road \\ Springfield, VA 22161 \\ Telephone: 703-605-6000 (1-800-553-6847) \\ TDD: $703-487-4639$ \\ Fax: 703-605-6900 \\ E-mail: info@ntis.fedworld.gov \\ Web site: http://www.ntis.gov/support/ordernowabout.htm
}

Reports are available to DOE employees, DOE contractors, Energy Technology Data Exchange (ETDE) representatives, and International Nuclear Information System (INIS) representatives from the following source:

Office of Scientific and Technical Information

P.O. Box 62

Oak Ridge, TN 37831

Telephone: 865-576-8401

Fax: 865-576-5728

E-mail: reports@adonis.osti.gov

Web site: http://www.osti.gov/contact.html

This report was prepared as an account of work sponsored by an agency of the United States Government. Neither the United States government nor any agency thereof, nor any of their employees, makes any warranty, express or implied, or assumes any legal liability or responsibility for the accuracy, completeness, or usefulness of any information, apparatus, product, or process disclosed, or represents that its use would not infringe privately owned rights. Reference herein to any specific commercial product, process, or service by trade name, trademark, manufacturer, or otherwise, does not necessarily constitute or imply its endorsement, recommendation, or favoring by the United States Government or any agency thereof. The views and opinions of authors expressed herein do not necessarily state or reflect those of the United States Government or any agency thereof. 


\title{
Protocols for Thermoluninescence and Optically Stimulated Luminescence Research at DOSAR
}

\author{
S. M. Bernal \\ University of Michigan \\ J. S. Bogard \\ Oak Ridge National Laboratory
}

October 2004

OAK RIDGE NATIONAL LABORATORY

Oak Ridge, Tennessee 37831-6285

managed by

UT-Battelle, LLC

for the

U.S. DEPARTMENT OF ENERGY

under contract DE-AC05-00OR22725 



\section{CONTENTS}

Page

LIST OF FIGURES $\ldots \ldots \ldots \ldots \ldots \ldots \ldots \ldots \ldots \ldots \ldots \ldots \ldots \ldots \ldots \ldots \ldots$

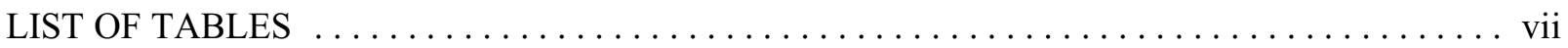

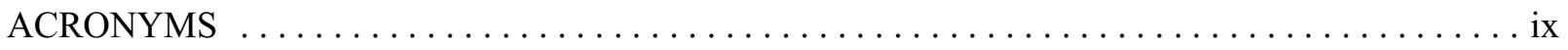

ACKNOWLEDGMENTS $\ldots \ldots \ldots \ldots \ldots \ldots \ldots \ldots \ldots \ldots \ldots \ldots \ldots \ldots \ldots \ldots \ldots \ldots \ldots$

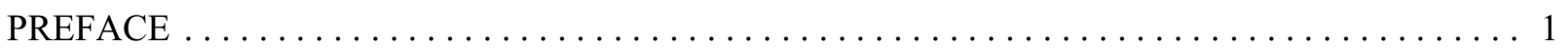

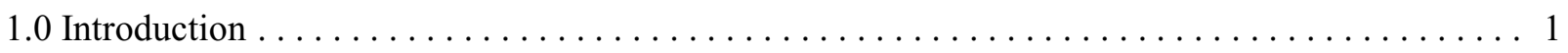

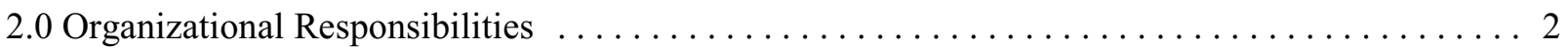

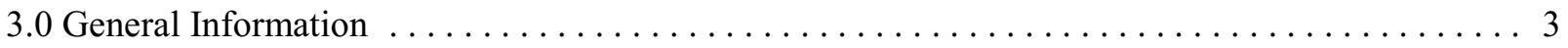

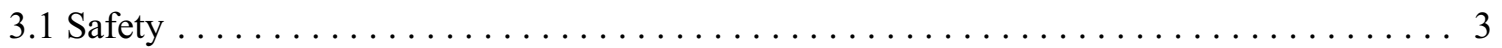

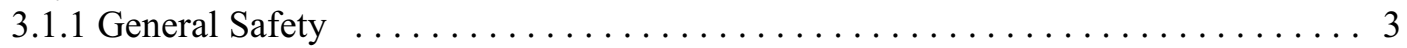

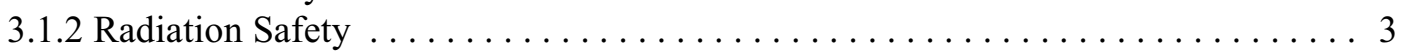

3.2 Qualifications, Responsibilities, and Training $\ldots \ldots \ldots \ldots \ldots \ldots \ldots \ldots \ldots \ldots$

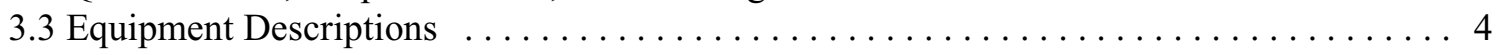

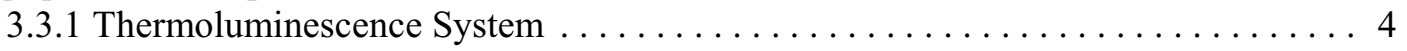

3.3.2 Optically Stimulated Luminescence System $\ldots \ldots \ldots \ldots \ldots \ldots \ldots \ldots$

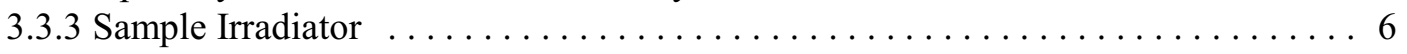

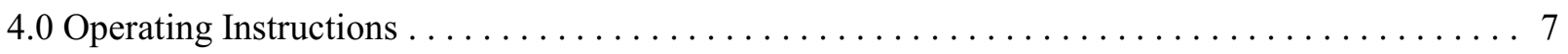

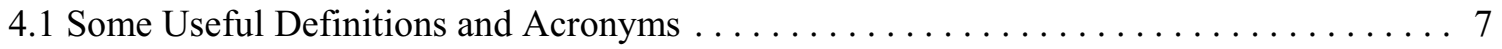

4.2 Daybreak 1100 TL Research System Operating Instructions … . . . . . . . . . . . 7

4.2.1 Daybreak Model 1100 TL Pre-Operational Procedure . . . . . . . . . . . . . . 8

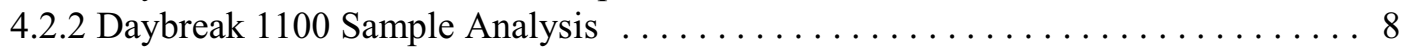

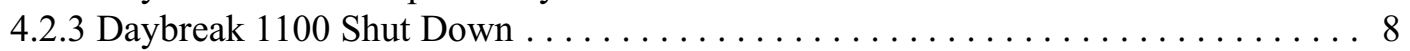

4.3 Daybreak 2200 OSL Reader System Operating Instructions $\ldots \ldots \ldots \ldots \ldots \ldots \ldots \ldots 11$

4.3.1 Daybreak Model 2200 OSL Pre-Operational Procedure . . . . . . . . . . . 11

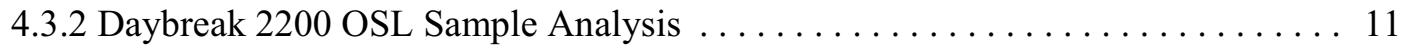

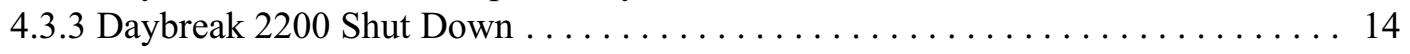

4.3.4 Recommended Response if Source Shutter Will Not Close . . . . . . . . . . 15

4.4 Model 801E Multiple Sample Irradiator Operating Instructions $\ldots \ldots \ldots \ldots \ldots \ldots \ldots 15$

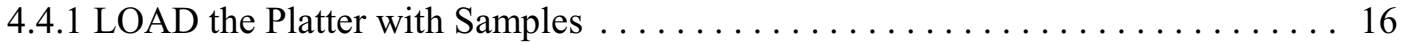

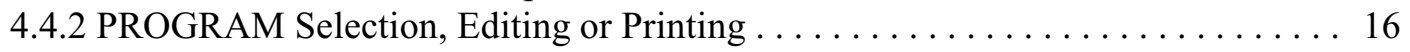

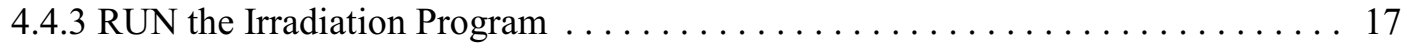

4.4.4 Recommended Response if Source Shutter Will Not Close . . . . . . . . . . . . 18

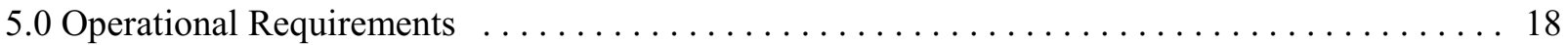

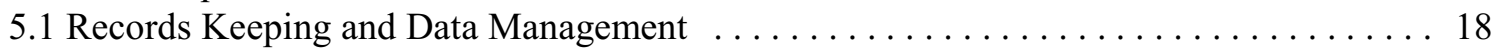

5.2 Planned Maintenance/Safety Checks/Calibrations . . . . . . . . . . . . . . . . . 19 


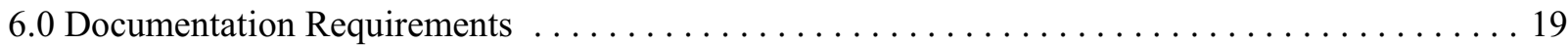

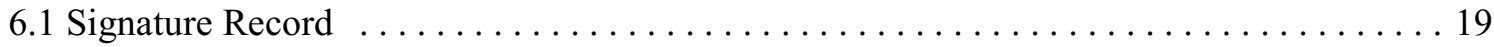

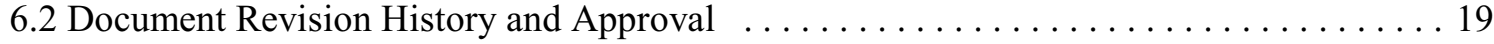

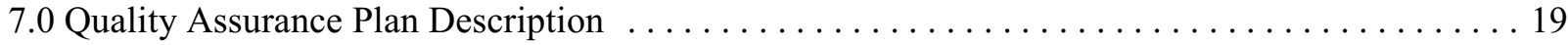

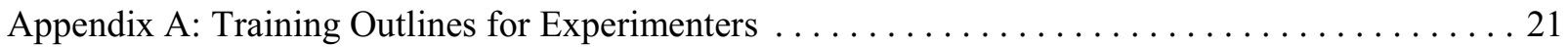

Appendix B: Schedule for Planned Maintenance, Safety Checks, and Calibrations . . . . . . . . 23

Appendix C: Procedures for Routine Maintenance, Safety Checks and Calibrations . . . . . . . 25

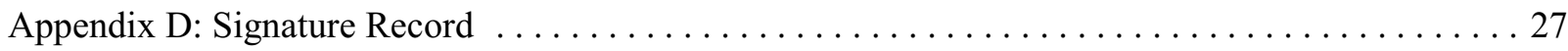

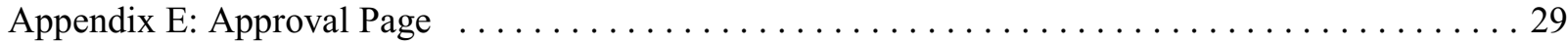




\section{LIST OF FIGURES}

\section{Figure}

Page

1 Daybreak Model 1100 TL system with cover and PMT assembly removed for

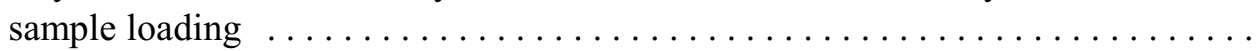

2 Lifting the sample platter from the Daybreak Model 2200 OSL system for sample

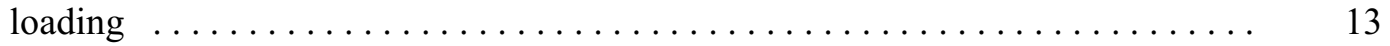

3 Sample loading station on the Daybreak Model 801E Irradiator. . . . . . . . 16 



\section{LIST OF TABLES}

\section{Table}

Page

$1 \quad$ Daybreak 1100 Preparatory Steps $\ldots \ldots \ldots \ldots \ldots \ldots \ldots \ldots \ldots \ldots \ldots \ldots$

2 Daybreak 1100 operation using a pre-existing script file $\ldots \ldots \ldots \ldots \ldots \ldots . \quad 10$

3 Daybreak 1100 operation without an existing script file $\ldots \ldots \ldots \ldots \ldots \ldots$

$4 \quad$ Shutting down the Daybreak $1100 \ldots \ldots \ldots \ldots \ldots \ldots \ldots \ldots \ldots \ldots \ldots \ldots \ldots \ldots \ldots$

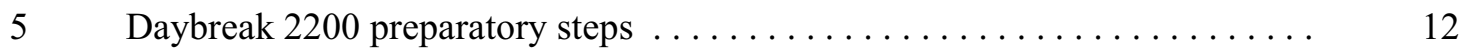

6 Daybreak 2200 operation using pre-existing script file $\ldots \ldots \ldots \ldots \ldots \ldots \ldots$

$7 \quad$ Daybreak 2200 operation without an existing script file $\ldots \ldots \ldots \ldots \ldots \ldots$

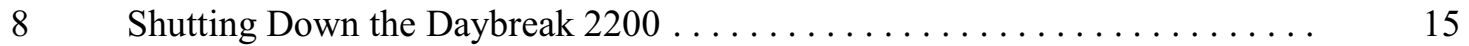





\section{ACRONYMS}

$\begin{array}{cl}\text { DOSAR } & \text { Dosimetry Applications Research } \\ \text { LCD } & \text { Liquid crystal display } \\ \text { LED } & \text { Light-emitting diode } \\ \text { LSD } & \text { Life Sciences Division } \\ \text { MOSL } & \text { Modulated OSL } \\ \text { ORNL } & \text { Oak Ridge National Laboratory } \\ \text { OSL } & \text { Optically stimulated luminescence } \\ \text { PI } & \text { Principal Investigator } \\ \text { PMT } & \text { Photomultiplier Tube } \\ \text { SBMS } & \text { Standards-Based Management System } \\ \text { TL } & \text { Thermoluminescence } \\ \text { UTK } & \text { University of Tennessee - Knoxville }\end{array}$





\section{ACKNOWLEDGMENTS}

The authors wish to thank R. L. Coleman and M. E. Murray for their assistance and advice in developing the capability for thermoluminescence and optically stimulated luminescence research at Oak Ridge National Laboratory. Instrumentation was procured through a University of Tennessee - Knoxville Center of Excellence Award to Jan Simek, Department of Anthropology, for use in collaborative research using these techniques to date archaeological artifacts. We are grateful to J. Michael Elam for fostering this collaboration and identifying worthwhile projects. This research was supported in part by an appointment to the Office of Civilian Radioactive Waste Management Graduate Fellowship program administered by the Oak Ridge Institute for Science and Education through an interagency agreement between the U.S. Department of Energy, with research being conducted at the Oak Ridge National Laboratory. 



\section{PREFACE}

The Life Sciences Division (LSD) of Oak Ridge National Laboratory (ORNL) has a long record of radiation dosimetry research at the Dosimetry Applications Research (DOSAR) facility complex. These facilities have been used by a broad segment of the research community to perform a variety of experiments in areas including, but not limited to, radiobiology, radiation dosimeter and instrumentation development and calibration, and materials testing in a variety of radiation environments. Collaborations with the University of Tennessee - Knoxville (UTK) have also led to important contributions in the area of archaeometry, particularly as it relates to the use of radiation dosimetry to date archaeological artificats.

This manual is to serve as the primary instruction and operation manual for dosimetric and archaeometric research at DOSAR involving thermoluminescence (TL) and optically stimulated luminescence (OSL). Its purpose is to (1) provide protocols for common practices associated with the research, (2) outline the relevant organizational structure, (3) identify the Quality Assurance plan, and (4) describe all the procedures, operations, and responsibilities for safe and proper operation of associated equipment. Each person who performs research at DOSAR using TL/OSL equipment is required to read the latest revision of this manual and be familiar with its contents, and to sign and date the manual's master copy indicating that the manual has been read and understood. The TL/OSL Experimenter is also required to sign the manual after each revision to signify that the changes are understood. Each individual is responsible for completely understanding the proper operation of the TL/OSL equipment used and for following the guidance contained within this manual. The instructions, protocols, and operating procedures in this manual do not replace, supersede, or alter the hazard mitigation controls identified in the Research Safety Summary ("Thermoluniscence/Optically Stimulated Luminescence Dosimetry") for this research, ORNL's Standards-Based Management System or any other official guidelines.

Changes to this report will be made as necessary to ensure that it remains accurate and current. The Principal Investigator (PI) for this research will review these Protocols in their entirety at least annually and certify that they are current by signing the Approval Page (Appendix D) of the master copy. If these Protocols are not up to date, the PI will initiate a revision to this document.

The master copy of the manual will be maintained in the office of the PI for this research, and a copy of the manual will be available at the location of the TL/OSL equipment.

\subsection{Introduction}

Thermoluminescence (TL) and optically stimulated luminescence (OSL) dosimetry research is conducted as part of the Dosimetry Applications Research (DOSAR) Program of the ORNL Life Sciences Division. The primary goals of the research are to investigate and refine radiation damage techniques for dating archaeological artifacts and to explore the fundamental nature of TL and OSL phenomena. The research is part of a collaborative effort with the University of Tennessee's Department of Anthropology.

Thermoluminescence and optically stimulated luminescence are analytical techniques that take advantage of the capacity of some materials for storing energy from their radiation environment and then releasing it as light when heated or stimulated with another light source having an appropriate wavelength. The radiation sensitivity of such materials taken from archaeological samples or from the natural environment must be determined to quantify the radiation field responsible for light emitted by the as-found sample. Radiation sensitivity is measured in these samples by exposure to known radiation fields, followed by TL or OSL analysis. 
Use of the TL/OSL equipment requires formal training to address safety issues associated with radiological and other hazards, along with task-specific training to ensure proper use of the equipment and design of experiments. Qualified Experimenters who have received the appropriate training are identified by the Principal Investigator in an Authorized User List, which is posted in the area where TL/OSL equipment is located.

The instructions, protocols, and operating procedures in this manual do not replace, supersede, or alter the requirements contained in the ORNL Standards-Based Management System (including those of the Radiological Protection Management System, Management System Description: Radiological Protection), or any other official safety guidance. Hazards identified in the research activities or use of equipment associated with TL/OSL research at DOSAR, and the controls implemented to mitigate these hazards, are described in the ORNL Research Safety Summary titled, "Thermoluniscence/Optically Stimulated Luminescence Dosimetry".

\subsection{Organizational Responsibilities}

The thermoluminescence/optically stimulated luminescence research effort is part of the Dosimetry Applications Research Program within the Advanced Biomedical Science and Technology Group of the Life Sciences Division at Oak Ridge National Laboratory. The following is a selected list of personnel positions and responsibilities directly related to TL/OSL research at DOSAR.

Life Sciences Division Director: Reviews and approves research operations through regularly reviewed Research Safety Summaries. Designates a divisional Safety Officer, Chemical Hygiene Program Officer, Radiation Control Officer and Training Officer to identify, implement and review required operational procedures, radiation and laboratory safety systems, and personnel training.

Principal Investigator (PI): Bears overall responsibility for the daily activities associated with TL/OSL research at DOSAR. Responsible for funding; management; personnel assignments; review and approval of operating protocols; work contracts; purchase orders; facility or equipment modifications; and for equipment and facility design and operation. Coordinates and corrects deficiencies found in safety reviews and inspections. Schedules research activities and equipment use. Reviews and updates the TL/OSL Protocol Manual as necessary and oversees task-based training.

Experimenter: Responsible for experimental design and setup. Submits operational requirements to the PI. Keeps a complete log of all experimental efforts and results.

LSD Training Officer: Identifies ORNL-required training for the PI and Experimenter, based on information in Research Safety Summaries and that provided by the PI. Assists in scheduling required training and maintains training records of the PI and Experimenter.

LSD Radiation Control Officer: Reviews operations within the Division for assurance that all radiological safety hazards associated with the research are identified, and that the PI has implemented appropriate controls to mitigate or minimize these hazards.

Operational Safety Services Division: Provides health physics support for the facility by ensuring that all personnel are assigned appropriate dosimetry and all radiation areas are properly surveyed and marked. Provides support for operators and experimenters during unusual events such as source transfers or emergency procedures. 


\subsection{General Information}

This section contains information about training requirements and safety responsibilities of ORNL employees and guests, especially as they apply to operation of TL/OSL equipment and facilities at DOSAR. Brief descriptions of the TL/OSL equipment are also provided.

\subsection{Safety}

Significant emphasis on safety at ORNL has resulted in a working environment where accidents are rare, and where those accidents that do occur are taken quite seriously. Anyone who observes an unsafe situation at ORNL, where there is a likelihood of injury to persons, environmental insult, or significant property damage, has the responsibility and authority to stop the work and request a safety review.

\subsubsection{General Safety}

It is the responsibility of each individual conducting TL/OSL research to ensure that all operations are carried out in a manner that minimizes the risk of accident or injury and that is consistent with safety practices outlined in the ORNL Standards-Based Management System and in Research Safety Summary, "Thermoluniscence/Optically Stimulated Luminescence Dosimetry".

\subsubsection{Radiation Safety}

Radiation safety is addressed in the ORNL Standards-Based Management System, Radiological Protection Management System, Management System Description: Radiological Protection, on the ORNL intranet. All personnel working with TL/OSL equipment should be familiar with the radiation sources used, the radiation fields they produce, and the safety systems in place to prevent potential exposure hazards. TL/OSL system operators are required to wear personnel radiation dosimeters assigned by the Operational Safety Services Division. Personnel dosimeters shall be worn on the front chest area outside of the clothing with the front of the badge facing outward.

Operations that require disassembly of the radioactive source housings or any activity that might result in a breach of shielding and exposure of personnel to the radiation sources shall be performed in the presence of an ORNL Radiological Control Technician (RCT). The RCT shall approve conditions under which the operation will be performed, monitor radiological conditions during the operation, and determine the suitability of the equipment for return to service at the operation's conclusion. ORNL's Radiation Source Control organization will also be informed of any such activity involving a Source Device ${ }^{1}$ and will be provided the opportunity to determine whether additional surveillance is required during the operation or before returning the Source Device to service.

If a source becomes jammed or stuck in the exposed position, the Principal Investigator will be notified and action will be taken as instructed in the section of the irradiation procedures which addresses equipment malfunctions.

\footnotetext{
${ }^{1}$ A Source Device is a sealed radioactive source with an associated radiation field of $1 \mathrm{rem} / \mathrm{h}$ or greater at $30 \mathrm{~cm}$. A ${ }^{90} \mathrm{Sr} /{ }^{90} \mathrm{Y}$ source associated with the Daybreak Model $801 \mathrm{E}$ Sample Irradiator and one associated with the Daybreak Model 2200 OSL system are classified as Source Devices.
} 


\subsection{Qualifications, Responsibilities, and Training}

The Principal Investigator (PI) and Experimenters constitute the two types of personnel performing TL and OSL experimental work at DOSAR. Qualifications of the Principal Investigator are determined by the Life Sciences Division as part of the selection process for that position. The PI functions as the program manager for TL/OSL projects, is responsible for developing and periodically reviewing the applicable Research Safety Summary (Research Safety Summary, "Thermoluniscence/Optically Stimulated Luminescence Dosimetry") for this research, and for development and periodic review of the TL/OSL research protocols set forth in this document. The PI also provides task-specific training for Experimenters; ensures, through consultation with the LSD Training Officer, that required ORNL training is identified and completed prior to performing work; and forwards documentation of completed training to the LSD Training officer. The PI is also responsible for TL/OSL equipment calibration and maintenance.

Qualifications for the Experimenter are determined by the PI, based on specific project goals. The Experimenter's qualifications should include the ability to operate the TL or OSL system which will be used for the project and any associated support equipment. Experimenters will be provided task-specific training by the PI or his designate. The Experimenter's responsibilities include TL or OSL sample analyses in support of project goals and timely entry of the corresponding information into the $\log$. TL/OSL Experimenters will receive ORNL-required training and task-specific training commensurate with project needs. A record of task-specific training will be generated by the PI or his designate and maintained by the LSD Training Officer. Task-specific training will include the material contained in Appendix A. The Experimenter is also required to review this protocol manual and equipment operating instructions. The final portion of the training will involve direct operations under the guidance and supervision of the PI or his designate.

\subsection{Equipment Descriptions}

Analytical equipment used in thermoluminescence and optically stimulated luminescence research at DOSAR includes commercially available (from Daybreak Nuclear and Medical Systems, Inc., Guilford, Connecticut) systems for thermoluminescence, optically stimulated luminescence, sample irradiation, and alpha-particle counting and spectroscopy. Operation of the TL and OSL systems is controlled by a dedicated personal computer and software supplied by the manufacturer. The sample irradiator and alpha counter have their own internal logic cirtuitry. Both the TL and OSL systems are supplied with pressurized nitrogen gas (analytical grade). The OSL system is also connected to a mechanical vacuum pump. Both the OSL system and sample irradiator contain sealed radioactive ${ }^{90} \mathrm{Sr} r{ }^{90} \mathrm{Y}$ sources for sample irradiations. The sample irradiator also contains a sealed radioactive source of ${ }^{241} \mathrm{Am}$ for sample irradiations. These radioactive sources are registered in the ORNL Radiation Source Inventory (RASIN) database and are tested periodically ${ }^{2}$ for containment integrity. The OSL reader and sample irradiator are classified as Source Devices ${ }^{3}$ because of the intense beta radiation fields generated by the ${ }^{90} \mathrm{Sr} /{ }^{90} \mathrm{Y}$ sources they house.

\subsubsection{Thermoluminescence System}

The Daybreak Model 1100 is a compact TL system measuring $27 \mathrm{~cm}$ wide, $29 \mathrm{~cm}$ deep, $14 \mathrm{~cm}$ high,

${ }^{2}$ Leak testing of sealed radioactive sources is currently required every 6 months by ORNL Procedures.

${ }^{3}$ Radiological Worker II (ORNL-specific) core radiological training is required for operation of these Source Devices. 
plus a 7.5-cm-diameter by 23 -cm-high photomultiplier tube (PMT) housing. It is controlled by a 'soft front panel' on the host computer. The utility TLCONSOLE is used for monitoring and exercising system operation, and includes an always-visible context-sensitive help window for sending commands. This 'front panel' display includes all status information in an easily read form. The 1100 continuously monitors operation by sending 22-byte packets of information to the host computer. (For reassurance, there is also a status display panel on the instrument.) With this arrangement, changes in system configuration may be made economically. A simplified set of status information is displayed while taking data, along with 'pushbuttons' and a real-time XY recorder-type display of data as they are received.

The sample changing mechanism of the 1100 uses a floating sweep arm to move samples on the turntable to and from a full-size heating plate. Both flat $(0.25-\mathrm{mm}$ thickness and above) and dished sample disks up to 0.375 -inch $(1.0-\mathrm{cm})$ diameter are accommodated. Fine grain and inclusion samples may both be analyzed due to the smooth motion afforded by precision DC micro-motors and worm gear drive. Dynamic braking motor control ensures positional reproducibility. The platter is removable for loading. Single samples may also be measured. The small volume of the glow oven (15 cm diameter by $1 \mathrm{~cm}$ deep) makes for fast evacuation. The glow oven is designed for use up to $700{ }^{\circ} \mathrm{C}$ for brief periods. The 1100 is configured with a serial interface (RS-232 at 9600 baud) for easy communication with any computer. Expansion capability has been built in as well, with the capability of adding up to 8 additional input/output ports (up to 128 input and output lines) within the system architecture, in addition to 3 analog inputs available on the board.

The 1100 is designed for use in dating geological samples, where automation is highly desirable. It is intended for use with the 801 multiple-sample alpha/beta irradiator, especially where irradiation times are long.

The single-grain laser scanner OSL adapter for the 2200 may also be used on the 1100 with a special lid.

\subsubsection{Optically Stimulated Luminescence System}

The Daybreak Model 2200 High-Capacity OSL Reader system uses light in the blue or infrared to stimulate the demotion electrons in the sample matrix that have been previously promoted to stable or metastable energy states. It then measures the light output resulting from this change of state.

The model 2200 has a single de-mountable $40-\mathrm{cm}$ platter that holds 60 samples. The total package dimensions are $50 \times 50 \mathrm{~cm}^{2}$ by $10 \mathrm{~cm}$ high (plus the photomultiplier barrel height). The top cover, upon which the OSL excitation array, PMT and irradiator are mounted, tilts up manually for access to the sample platter. The sample platter may be loaded in place, or it may be removed and loaded elsewhere. The sample moves from one stage to the next in sequence under control of the software running on the host computer. A mechanical shutter protects the PMT from ambient light when the cover is up, to minimize recovery time. The top cover is locked in place during the measurement cycle.

The sample is raised from the platter during readout by a temperature-controlled stage (thermoelectric finger), and light baffles from above and beneath touch the platter to isolate the inactive samples adjacent to the sample under test from any exposure to the blue $(480-\mathrm{nm})$ or IR (880-nm) excitation light.

Because the size of the 2200 platter device is so large, there is sufficient room to place a beta 
irradiator without increased dark counts from bremsstrahlung. A sealed $100-\mathrm{mCi}{ }^{90} \mathrm{Sr} /{ }^{90} \mathrm{Y}$ source (ORNL Radiation Source Inventory number SR-90-5250) housed in the OSL reader system has a nominal activity of $100 \mathrm{mCi}\left(3.7 \times 10^{9} \mathrm{~Bq}\right)$, as reported by the manufacturer on 13 December 2002 . The sample is moved up by an elevator into a shielded irradiation space (15-mm source-to-sample distance) in order to prevent or minimize exposure to adjacent samples. (Adjacent samples receive less than $0.02 \%$ of the exposed sample's radiation dose.)

The 2200 has thermoelectric sample temperature control $\left(0-140^{\circ} \mathrm{C}\right)$ and preheat (ambient to $500{ }^{\circ} \mathrm{C}$ ) stations for the beta irradiator. The separate preheat stage is included to reduce cycle time without need of a cooling gas. The controller of the 2200 permits use of a general temperature profile for the irradiation sample temperature. This makes it possible to irradiate at elevated temperature (up to $\left.300{ }^{\circ} \mathrm{C}\right)$.

The 2200 OSL Reader may be used with the IRIS4 Quad Detector, which is able to measure light in the 250-620 nm range, and with the Scanned Laser Exciter. The Scanned Laser Exciter permits irradiation and heating of many grains at once, and individual readout for dosimetry and grain characterization.

\subsubsection{Sample Irradiator}

The $801 \mathrm{E}$ multiple sample irradiator is capable of irradiating up to 30 disks or aliquots to sealed sources of both alpha and beta radiations. Features of this countertop irradiator include use of a large display (two lines of 40 characters, red backlit negative-image LCD that dims automatically with changing ambient light level) making navigation through the function menu possible using cursor keys. A realtime clock/calendar makes it possible to compute decay for the radionuclides housed in the 801E's two irradiators, and an EEPROM stores multiple irradiation programs. There also is a serial channel, either RS-232 or RS-485 (optional) for connecting multiple irradiators (and alpha counters) to a single serial port on an external computer for exchange of status information and downloading irradiation programs. There is nonvolatile storage for up to 10 irradiation programs, up to 10 sets of dose rates to different materials, easy editing, and irradiation either by time of exposure or by set dose to be delivered (using decay-corrected dose rates for the particular material irradiated). Battery-backed random access memory stores the current state for protection against power failure, and operation resumes without interruption (but with a printed log of failure time) when power is returned. Security features include possibility of a password to be entered before operation, and inaccessibility of irradiators except though the bottom. The exposure rate at the surface of the front panel, with a $100-\mathrm{mCi}$ Sr-90 ceramic beta source [ORNL Radiation Source Inventory number SR-90-5251 with a nominal activity of $100 \mathrm{mCi}\left(3.7 \times 10^{9} \mathrm{~Bq}\right)$, as reported by AEA Technology on 13 December 2002] loaded, is only $10 \mu \mathrm{R} / \mathrm{hr}$. The worst case for any accessible surface ( $8 \mathrm{~cm}$ from the rear of the case, top center) is $500 \mu \mathrm{R} /$ hour, and this is reduced to $90 \mu \mathrm{R} /$ hour at a position $30 \mathrm{~cm}$ above the surface. ${ }^{4}$

A plated ${ }^{241} \mathrm{Am}$ alpha source (ORNL Radiation Source Inventory number AM-241-5252) housed in the Model $801 \mathrm{E}$ Irradiator has a nominal activity of $241 \mu \mathrm{Ci}\left(8.92 \times 10^{6} \mathrm{~Bq}\right)$, as reported by the manufacturer (AEA Technology) on 20 December 2002.

${ }^{4}$ Radiation exposure rates in the vicinity of the Model $801 \mathrm{E}$ Irradiator with a $100-\mathrm{mCi}{ }^{90} \mathrm{Sr}$ source installed were provided by Daybreak Nuclear and Medical Systems, Inc. 


\subsection{Operating Instructions}

Operating instructions for typical sample analyses utilizing the Daybreak TL and OSL reader systems and for calibrated irradiation of samples using the sample irradiator are provided in this section. These instructions provide a synthesis of the material contained in the Daybreak operating manuals, which should be consulted for a more sophisticated understanding of these systems and for tailoring experiments with different requirements.

\subsection{Some Useful Definitions and Acronyms}

Thermoluminescence (TL) - refers to the light emission that occurs during heating of a sample that has already absorbed some energy from radiation.

Optically Stimulated Luminescence (OSL) - refers to the luminescence emitted from an irradiated insulator or semiconductor during exposure to light. Light stimulation is constant in intensity.

Modulated OSL (MOSL) - A method of OSL in which the luminescence is emitted from an irradiated insulator or semiconductor by increasing the stimulation light intensity from zero to a maximum value.

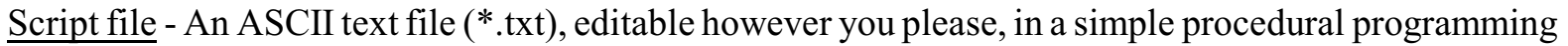
language, with variables, list arrays, procedure definitions, and loops. It can be used to define virtually any method or issue any command that you want carried out by the 1100 or the 2200 for the purposes of measuring or testing. A script file is written using the formal language and syntax described in the TLApplic manual starting on page 26.

Method definitions - A script file which describes some basic operations applied to a group or groups of aliquots (or disks) in a non-quantitative way. It defines the method abstractly.

Laboratory specific measurement template - A Script file in which a template is modified to meet the requirements of particular samples dated in the laboratory and form 'executable' scripts that are used either to directly control the whole process of measurements or to generate the batch program(s) necessary to achieve the same goal.

FLConsole - Abbreviation for the FirstLight Console Windows software which is used to operate the Daybreak models 1100 and 2200. The experimenter uses the FirstLight Console to control the actions of both models - either by loading and executing a user specified script file, or by issuing direct commands using the FirstLight interface. There is also a FirstLight Script Editor program for editing script files as well as a FirstLight Editor program for performing data analysis.

TLApplic - The DOS version of the operational software for the 1100 and the 2200 . This program is the alternative to FLConsole, and allows modification of the 'TLinfo.dta' parameter file, which the windows software does not allow. For details, see the Daybreak software manual (TLApplic v. $4.30 \& 4.40$ ).

\subsection{Daybreak 1100 TL Research System Operating Instructions}

The instructions below give an outline of how to perform TL measurements on the Daybreak model 1100. For more information on the 1100 hardware, as well as the associated FirstLight and TLApplic software data analysis capabilities, the experimenter is strongly encouraged to read the Daybreak manuals. 
In the following, the words 'disk(s)', 'aliquot(s)' and 'sample(s)' are used interchangeably.

\subsubsection{Daybreak Model 1100 TL Pre-Operational Procedure}

Samples are loaded and the sample chambers are evacuated, then purged with nitrogen prior to applying high voltage to the photomultiplier tube.

A stepwise pre-operational procedure is provided in Table 1.

NOTE: Ensure that the high voltage is turned off whenever loading or unloading samples, or whenever lifting the PMT off the 1100.

\subsubsection{Daybreak 1100 Sample Analysis}

Measurements can be performed either by loading an executable script file into the FLConsole operational software (see Option 1 below), or by using the FLConsole software window to directly give operational instructions to the 1100 (Option 2). For simplicity, only the Windows software (FLConsole) will be addressed here, but for details on the TLApplic operational software please refer to the software manual for directions.

Option 1. A script file may be written beforehand (see section 'TLApplic v $4.30 \& 4.40$ ' of the Daybreak software manual page 26). This file can be executed using the stepwise procedure provided in Table 2 .

Detailed instructions on how to write a script file, or the various script files which are already available for use, are contained in the 'Daybreak Systems for TL Research' software binder behind the 'TLAPPLIC v $4.30 \& 4.40$ ' tab. Script files for the 1100 already included in the software package are 'R_Dose', 'TL_slide', 'TL_slide_n', and 'TL_additive'. Each of these files performs measurements using a specific protocol to meet the data analysis requirements for that method (see pages 17-25 of the software manual). Pre-existin script files should be reviewed for suitability before use.

Option 2. No script file is necessary if the FirstLight console window is used to enter parameter values for glow curve acquisition (for a single disk or a range of disks). A stepwise procedure is provided in Table 3.

\subsubsection{Daybreak 1100 Shut Down}

The nitrogen supply is secured and high voltage to the photomultiplier tube is shut off prior to deenergizing the unit. The stepwise shut-down procedure is provided in Table 4. 
Table 1. Daybreak 1100 Preparatory Steps

\begin{tabular}{|c|c|}
\hline Step & Action \\
\hline 1 & Make sure that the serial cord from the 1100 TL machine is connected to the serial port on the computer. \\
\hline 2 & Verify that the 1100 is plugged into the wall, hooked up to the nitrogen line and the vacuum. \\
\hline 3 & Turn on the 1100 by flipping the switch located behind the machine on the right-hand side. \\
\hline 4 & Turn on the red lights and turn off fluorescent and incandescent room lights, if required. \\
\hline 5 & $\begin{array}{l}\text { Grasping firmly onto the PMT situated on top of the } 1100 \text { carefully lift upwards until the PMT together } \\
\text { with the cover is lifted off the } 1100 \text {, as shown in Figure } 1 \text {. You will now be able to see the sample platter } \\
\text { (with } 19 \text { sample locations) as well as the arm which moves the samples on and off the heating planchet. }\end{array}$ \\
\hline 6 & Set the PMT aside, minimizing unnecessary exposure to room light. \\
\hline 7 & $\begin{array}{l}\text { Carefully lift the sample transfer arm so that it's pointing to the ceiling. Be careful not to jostle the arm as } \\
\text { it may fall back down and disturb samples on the platter. }\end{array}$ \\
\hline 8 & Load samples onto the platter. \\
\hline 9 & $\begin{array}{l}\text { Once you're finished loading samples, use both hands to carefully replace the PMT /lid. Make sure to line } \\
\text { up the pin sticking out of the lid with the corresponding notch on the base of the } 1100 \text {. }\end{array}$ \\
\hline 10 & $\begin{array}{l}\text { Double-click on the 'FLConsole' icon located on the Windows 'start' menu. This will open the Windows } \\
\text { version of the software that is used to control the } 1100 \text { or the } 2200 \mathrm{TL} / \mathrm{OSL} \text { machines. It may say } \\
\text { 'FirstLight Console } 2200 \text { System' across the top of the window. Ignore this. }\end{array}$ \\
\hline 11 & $\begin{array}{l}\text { Click on 'settings' at the top of the window, and then select 'reader port', then 'port settings'. A window } \\
\text { should open up that says 'Setup'. Click on the down arrow next to 'Port' and select COM1. Click OK. }\end{array}$ \\
\hline 12 & Under 'Settings' select '1100 System' \\
\hline 13 & $\begin{array}{l}\text { Click on 'Serial port', then 'Open port'. You should now see the numbers on the right under 'Data' start to } \\
\text { fluctuate. }\end{array}$ \\
\hline 14 & Turn on the vacuum located under the 1100 inside the cabinet. \\
\hline
\end{tabular}

If you go back to the FirstLight Console window on your computer, you'll notice that the screen is separated into four main areas which are (left to right): Commands, Data, Status, and Errors. Before taking data you must instruct the machine to do the following:

Open up the bleed and main valves to create a vacuum in the sample chamber. This is done by entering the command 'V1' in the field marked 'Send command to reader' and hitting Enter. The bleed valve should then open on the machine, and when the vacuum is high enough, the main valve will also open.

Supply nitrogen to the machine by opening the pressurized tank's shutoff valve and setting the regulator to 20 pounds per square inch.

17 Close the valves by entering 'V0' in the same field as before when the vacuum has gone below $50 \mu \mathrm{m} \mathrm{Hg}$.

18 Enter the command 'P1' to turn on the purge gas (i.e. nitrogen).

19 Turn off the vacuum in the cabinet, if you like.

20 Enter the command 'H1' to turn on the high voltage to the PMT.

NOTE: Ensure that the high voltage is turned off whenever loading or unloading samples, or whenever lifting the PMT off the 1100 . 


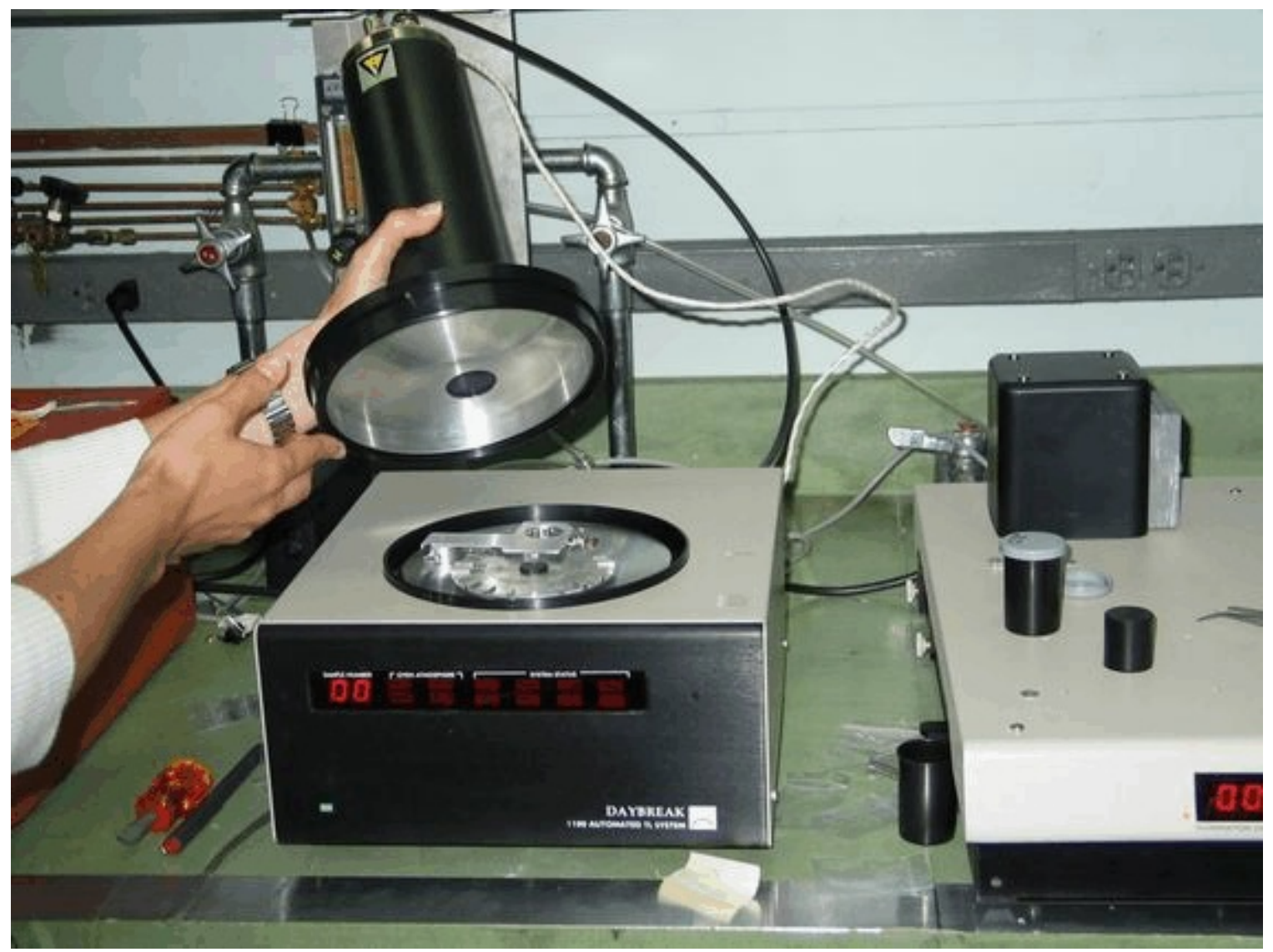

Figure 1. Daybreak Model 1100 TL system with cover and PMT assembly removed for sample loading.

\section{Table 2. Daybreak 1100 operation using a pre-existing script file}

\begin{tabular}{l} 
Step Action \\
\hline $1 \quad$ In the FirstLight window, select 'Take data' and then 'Take script'. \\
The program will then ask you to 'load the reader's system data'. Select 'System' and then 'Load system \\
info'. The reader's system data is located in the TLinfo.dta file located in the following directory \\
'C: $:$ DAYB $\backslash$ Dayb1100 ${ }^{\prime}$ '. Select this file and then click 'Open'. \\
Open the script file you want executed by selecting 'File' then 'Open'. Browse the directory for your file \\
and then click on 'Open'. The text of your script file should now be visible. Look it over to make sure it \\
is correct. \\
Now click on 'Script' at the top of the FLConsole window, and then select 'Interpret Script'. The program \\
will check for errors and alert you if it finds any. If no errors are found, select 'Script' again and then \\
'Execute script'. The 1100 should now start making measurements according to the commands contained \\
in your executable script file. \\
If you have not included the appropriate command in the script file the software will write all your data to \\
a text file called 'default.txt'. If you would like to change the name of the file you can either do it after the \\
measurements are taken, or you can enter the command 'OPEN 'datafilename' 'in the script file. This \\
command will tell the software to create a text file with the name datafilename in the default data directory. \\
This file ('datafilename.txt') can be imported to Excel to carry out calculations or analysis. Other data \\
analysis methods are also available - see page 17-25 of the software manual. Each Experimenter will have \\
a dedicated subdirectory in which to permanently store data files.
\end{tabular}


Table 3. Daybreak 1100 operation without an existing script file

Step Action

In the FirstLight Console Window on your computer select 'Take data' and then 'Take TL'. You should now be able to enter in your instructions for glow curve acquisition. Parameters such as ramp rate, maximum temperature, maximum temperature hold time, cooling temperature, and preheat temperature and duration, for a specific disk or range of disks can be set to desired values. In the box labeled 'Text

1 Info' you may enter descriptive sentences describing your measurements or the protocol followed with your samples. Under 'Data file name' you may also enter the name of the data file that you want created to store the results of your measurements, as well as the directory your would like that file saved to. (Each Experimenter will have a dedicated subdirectory in which to store data files.)

2 Click 'Ok' to accept your entries. The 1100 should now begin measurements.

Table 4. Shutting down the Daybreak 1100

\begin{tabular}{ll} 
Step & Action \\
\hline 1 & $\begin{array}{l}\text { Shut off nitrogen flow from the pressurized tank by closing the shutoff valve on top of the tank. Turn the } \\
\text { pressure regulator counterclockwise at least two rotations to prevent a pressure surge when the shutoff } \\
\text { valve is next turned on. }\end{array}$ \\
2 & Enter 'H0' to turn off the high voltage to the PMT if it is not already off. \\
3 & Unload samples if desired \\
4 & Turn off the 1100.
\end{tabular}

\subsection{Daybreak 2200 OSL Reader System Operating Instructions}

The instructions below give an outline of how to perform OSL measurements on the Daybreak model 2200. For more information on the 2200 hardware, as well as the associated FirstLight and TLApplic software data analysis capabilities, the experimenter is strongly encouraged to read the Daybreak manuals.

In the following, the words 'disk(s)', 'aliquot(s)' and 'sample(s)' are used interchangeably.

\subsubsection{Daybreak Model 2200 OSL Pre-Operational Procedure}

Samples are loaded and the analytical chamber is purged with nitrogen gas prior to supplying high voltage to the photomultiplier tube. A stepwise procedure is provided in Table 5.

\subsubsection{Daybreak 2200 OSL Sample Analysis}

Measurements can be performed using the Daybreak 2200 OSL system either by loading an executable script file into the FLConsole operational software (see Option 1 below), or by using the FLConsole software window to directly give operational instructions to the 2200 (Option 2).

Option 1. Write a script file beforehand (see section 'TLApplic v $4.30 \& 4.40$ ' of the Daybreak 
software manual page 26). This file can be executed using the stepwise procedure in Table 6 .

Table 5. Daybreak 2200 preparatory steps

\begin{tabular}{|c|c|}
\hline Step & Action \\
\hline 1 & Make sure that the serial cable from the 2200 OSL system is connected to the serial port on the computer. \\
\hline 2 & $\begin{array}{l}\text { Verify that the } 2200 \text { is plugged into a power source as well as hooked up to the nitrogen line. Note that } \\
\text { the } 2200 \text { is designed to stay on at all times, and therefore does not have a power switch. }\end{array}$ \\
\hline 3 & Turn on the red lights and turn off fluorescent and incandescent room lights, if required. \\
\hline 4 & $\begin{array}{l}\text { The lid of the } 2200 \text { supports the PMT and the irradiator. On the right hand side near the front you will see } \\
\text { a black knob labeled 'Shutter closed/Lid unlock' on one end and 'Shutter open/Lid lock' on the other. Slide } \\
\text { the knob to the 'Shutter closed/Lid unlock' position. This will close the light shutter on the PMT and } \\
\text { unlock the lid so that you can open it. }\end{array}$ \\
\hline 5 & Grasp the edges of the lid under the front panel display and lift. \\
\hline 6 & $\begin{array}{l}\text { You should now be able to see the sample platter. The platter is generally loaded while removed from the } \\
2200 \text {, as in Figure 2, but it can also be loaded in place. During loading you may turn on the red LEDs in } \\
\text { back of the front panel with the switch under the front panel display at the bottom edge of the cover. } \\
\text { Remember to turn them off before closing the cover. }\end{array}$ \\
\hline 7 & $\begin{array}{l}\text { Loading is best done in the zero position (sample position } 0 \text { at the detector sample stage -right rear of the } \\
\text { instrument) by using the ' } \mathrm{J} 0 \text { ' or ' } \mathrm{Z} \text { ' command (this is done on power on or reset, after a measurements or } \\
\text { by using the commands listed in the FirstLight Console window). }\end{array}$ \\
\hline 8 & After loading all samples close the lid and slide the black front knob to 'Shutter open/Lid lock'. \\
\hline 9 & $\begin{array}{l}\text { Double-click on the 'FLConsole' icon located on the Windows 'start' menu. This will open the Windows } \\
\text { version of the software that is used to control the } 1100 \text { or the } 2200 \text { TL/OSL machines. }\end{array}$ \\
\hline 10 & $\begin{array}{l}\text { Click on 'settings' at the top of the window, and then select 'reader port', then 'port settings'. A window } \\
\text { labeled 'Setup' should open up. Click on the down arrow next to 'Port' and select 'COM1'. Click OK. }\end{array}$ \\
\hline 11 & Under 'Settings' select '2200 System'. \\
\hline 12 & $\begin{array}{l}\text { Click on 'Serial port', then 'Open port'. You should now see the numbers on the right under 'Data' start to } \\
\text { fluctuate. }\end{array}$ \\
\hline 13 & $\begin{array}{l}\text { Supply nitrogen to the machine by opening the pressurized tank's shutoff valve and setting the regulator } \\
\text { to } 20 \text { pounds per square inch. Check to be sure the second shutoff valve on the copper line behind the } 2200 \\
\text { is open. }\end{array}$ \\
\hline \multicolumn{2}{|r|}{$\begin{array}{l}\text { If you go back to the FirstLight Console window on your computer, you'll notice that the screen is separated into four } \\
\text { main areas which are (left to right): Commands, Data, Status, and Errors. Before taking data you must instruct the } \\
\text { machine to do the following: }\end{array}$} \\
\hline 14 & Enter the command "P1" in the 'Send Command to Reader' window to turn on the purge gas (i.e. nitrogen). \\
\hline 15 & Enter the command "H1" to turn on high voltage to the PMT. \\
\hline
\end{tabular}




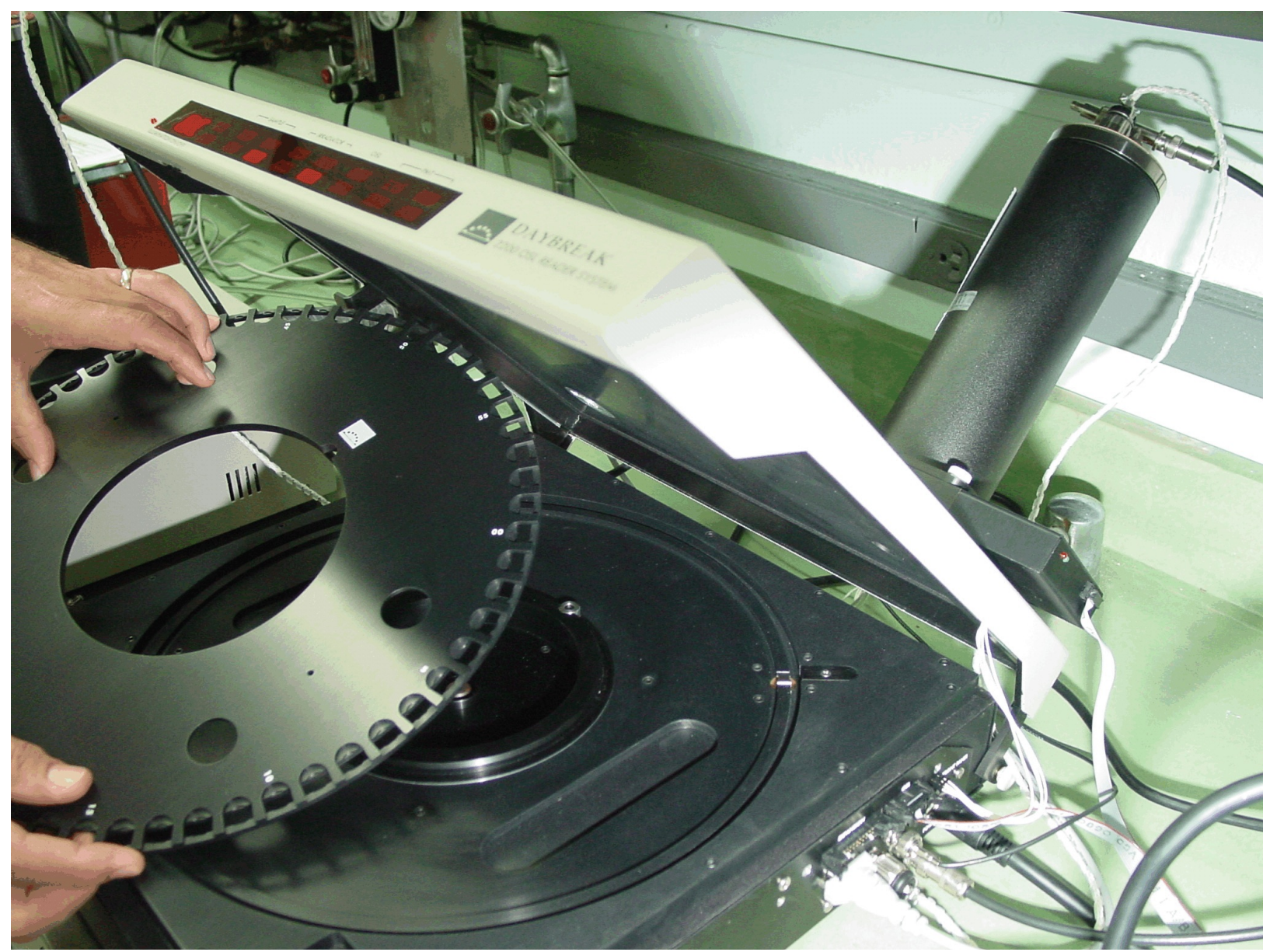

Figure 2. Lifting the sample platter from the Daybreak Model 2200 OSL system for sample loading.

Detailed instructions on how to write a script file, or the various script files which are already available for use, are contained in the 'Daybreak Systems for TL Research' software binder behind the 'TLAPPLIC v $4.30 \& 4.40$ ' tab. Included with the software (at path C: $\backslash D A Y B \backslash P R O G R A M S$ for OSL and TL $\backslash$ OSL) are Script files designed to carry out the following OSL dating methods: Single aliquot additive dose method (SAA.txt), and the Single aliquot regenerative dose method (SAR.txt and SARSCRPT.txt). Each of these files performs measurements using a specific protocol to meet the data analysis requirements for that method (see pages 17-25 of the software manual). However, all files should be examined before use for incorrect or unwanted experimental set up.

Option 2. No script file is necessary if the FirstLight console window is used to enter parameter values for OSL or MOSL data acquisition (for a single disk or a range of disks). This method is best for simple measurements or for operational testing. The stepwise procedure is shown in Table 7. 


\begin{tabular}{ll} 
Step & Action \\
\hline 1 & $\begin{array}{l}\text { Shut off nitrogen flow from the pressurized tank by closing the shutoff valve on top of the tank. Turn the } \\
\text { pressure regulator counterclockwise at least two rotations to prevent a pressure surge when the shutoff } \\
\text { valve is next turned on. }\end{array}$ \\
2 & Enter 'H0' to turn off the high voltage to the PMT if it is not already off. \\
3 & Unload samples if desired.
\end{tabular}

\subsubsection{Recommended Response if Source Shutter Will Not Close}

A shutter disk (aluminum and lead composite for minimum brehmsstrahlung production) is turned by a rotary solenoid when energized so that an aluminum-lined aperture allows the source to irradiate the sample. A mercury tilt switch interlock allows the shutter to open only when the lid is down; a return spring closes the shutter when power is removed.

A shutter which will not close by command from the front panel can be closed manually by disassembling the instrument's case and gaining access to the top of the irradiator. (Note that, even with the shutter in open position, the only region actually exposed to beta radiation is the sample which is positioned directly below the source, as long as the source shield assembly remains uncompromised.)

Any condition that requires disassembly or modification of the source shield must be performed in the presence of an ORNL RCT, who shall approve conditions under which the operation will be performed, monitor radiological conditions during the operation, and determine the suitability of the equipment for return to service at the operation's conclusion.

\subsection{Model 801E Multiple Sample Irradiator Operating Instructions ${ }^{5}$}

The Daybreak $801 \mathrm{E}$ will expose up to 30 samples sequentially to alpha and beta radiation. The radiation type and exposure time (up to 99999.9 seconds) are individually programmable for each sample position through a flexible, interactive data entry procedure with prompt messages. There is no power switch on the $801 \mathrm{E}$; power is supplied continuously as long as the instrument is plugged into a 120-VAC outlet.

The $801 \mathrm{E}$ does not normally communicate with a host computer, but is operated from the front panel, which has a red backlit liquid crystal display (LCD), status-indicating light-emitting diodes (LEDs) (green for 'safe/shutter closed'; red for 'shutter open') for the two irradiators, and two key pads. The left key pad consists of a group of six keys for menu navigation, including two arrow keys for cursor movement, $<$ ENTER $>$ and $<$ CLEAR $>$ keys, a $<$ STOP/ABORT $>$ key to interrupt irradiation, and a paper advance key for the printer. The right-hand key pad is for data entry and includes the numerals $0-9$, the decimal point, and a backspace key $<$ BS $>$.

The operations menu is navigated using the three keys $\leftarrow, \rightarrow$ and $<$ ENTER $>$. The $\leftarrow$ and $\rightarrow$ keys are used to move between menu choices. A brief description (prompt) appears on the lower line of the display describing each choice. Choices which may be highlighted by the left and right arrow keys $\leftarrow$ and $\rightarrow$ are

\footnotetext{
${ }^{5}$ Excerpts from the Daybreak Model 801E Operating Manual.
} 
LOAD, PROGRAM, RUN and OPTIONS. Pressing $<$ ENTER $>$ selects the choice. A description of each choice is shown on the second line of the display:

LOAD Load the platter with samples.

PROGRAM Select, edit, or print the irradiation program.

RUN Run the program.

OPTIONS Enter the date and time.

\subsubsection{LOAD the Platter with Samples}

Use the arrow keys to highlight LOAD, and press $<$ ENTER $>$ when finished. The display shows the platter position that is at the load door on the front apron of the 801E, shown in Figure 3. The platter has a 0.5 -mm-deep well for 10 -mm-diameter sample disks or planchets at each position. There is a deeper well in the center of each position for 3-mm-square dosimeter chips as well.

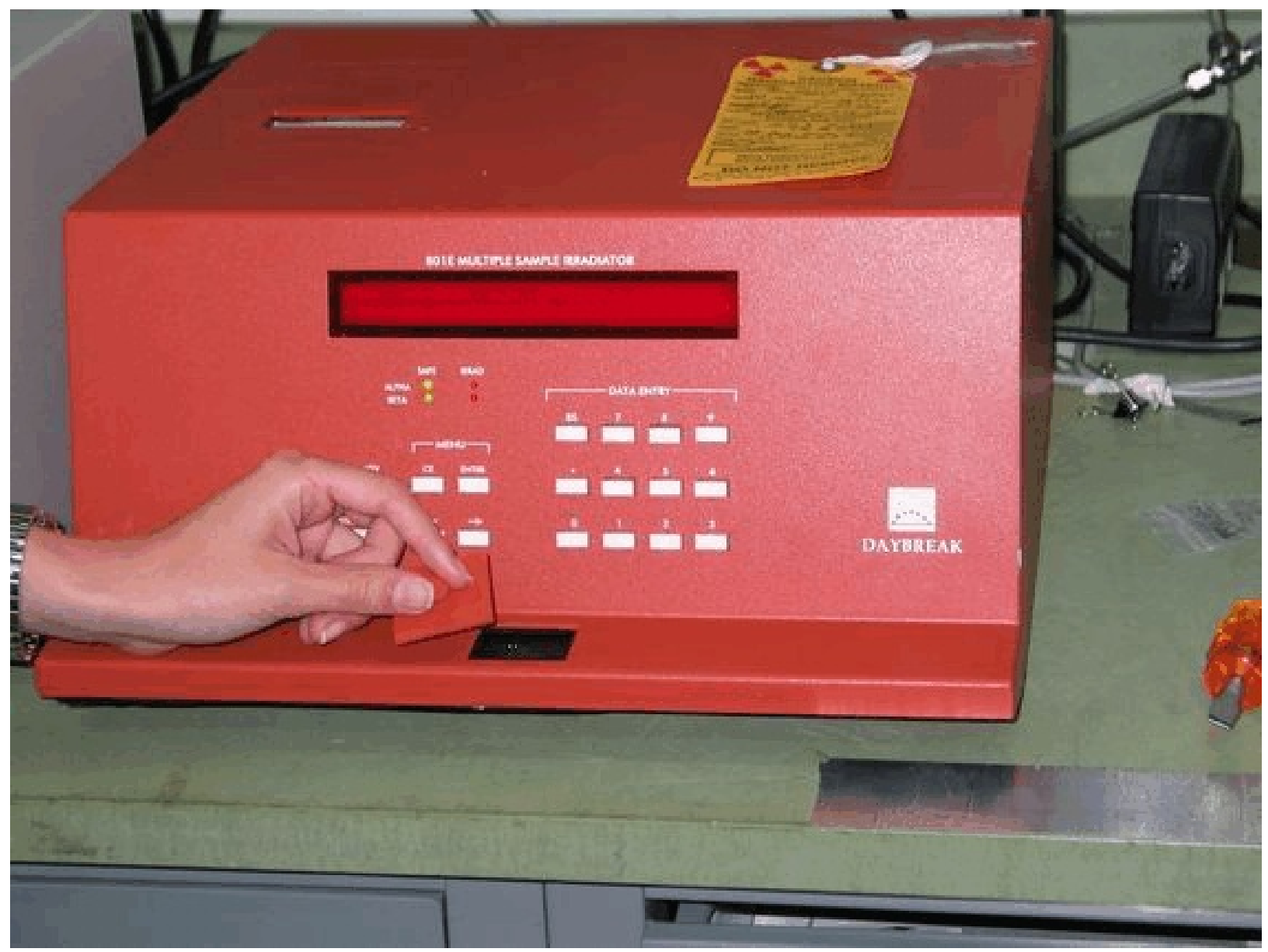

Figure 3. Sample loading station on the Daybreak Model 801E Irradiator. Note the red LED readout and the programming keys on the front panel.

\subsubsection{PROGRAM Selection, Editing or Printing}

This function allows the creation of a new irradiation program, or editing of the previously stored program. The Program menu choices can apply to each sample position individually and consist of: 
NEW Create a new program.

Selecting $N E W$ overwrites the previous program and creates a display that reads:

$$
\begin{array}{ll}
00 \quad 0000.0 \quad \text { Beta } & \text { Next } \quad \text { Done } \\
\text { Position } \leftarrow \text { or } \rightarrow & \text { OK }=<\text { ENTER }>
\end{array}
$$

Use the arrow keys to select a starting sample position (00 through 29) in the first field, then press $<$ ENTER $>$ to go on the time setting in the second field, where the prompt is

$$
\text { Time in seconds } \quad<\text { ENTER }>=\text { done. }
$$

In revisions 0 and 1, you must either enter leading zeroes or use the $\rightarrow$ key to get to the first significant digit. Enter the time up to 99999.9 seconds. The cursor keys and $<$ BS $>$ key allow you to navigate the time field. You actually are overwriting the data shown in the field $(00000.0$ seconds is the default - you can skip positions if you want). Press $<$ ENTER $>$ to record the exposure time and move the cursor to Beta.

Use the left and right arrow keys to toggle between the two irradiator choices, 'Beta' $\left({ }^{90} \mathrm{Sr} /{ }^{90} \mathrm{Y}\right)$ or 'Alpha' $\left({ }^{241} \mathrm{Am}\right)$, and press $<$ ENTER $>$ when correct.

The cursor is now positioned at Next. Press $<$ ENTER $>$ to program the irradiation for the next sample position, or use the $\rightarrow$ key to move the cursor to Done.

Press $<$ ENTER $>$ with the cursor at Done to end the program.

EDIT Modify the current program.

This menu choice allows editing of specific program settings without overwriting the whole program. Selections are the same as in $N E W$.

PRINT Prints the current irradiation program.

RETURN Return to main menu.

\subsubsection{RUN the Irradiation Program}

Choose $R U N$ to execute the irradiation program. The beta irradiator is half way around the platter from the load door, so if sample position number 0 is under the beta irradiator, position 15 is at the load door and " 15 " appears in the display's lower right. The alpha irradiation is $6 / 10$ of the way around, so 0 under the alpha irradiator puts position 18 at the load door. The display shows the sample number being irradiated (alpha or beta), the total time to be exposed, and the time remaining. The position under the load door is displayed at the lower right.

Nonvolatile memory containing system variables is updated ten times per second when running. If there is a power failure, the irradiation will resume when the power is restored and print a message that a failure has occurred. The program remains intact.

To interrupt the execution of $R U N$, press the $S T O P / A B O R T$ key at any time. The active shutter will close, and a menu will be displayed, giving you the choice (by use of the cursor and $<$ ENTER $>$ keys) 
of resuming operation, editing the program, loading, or aborting altogether. Program editing or sample loading may be performed while the irradiation is stopped, after which RESUME may be selected from this menu to continue the irradiation program from the point of interruption.

\subsubsection{Recommended Response if Source Shutter Will Not Close}

Return springs on the rotary solenoids that open the radioactive source shutters are sufficiently strong to keep the shutters firmly closed in all circumstances when the solenoids are not energized. A lead counterweight is located opposite each shutter to prevent unpowered shutter opening if the instrument is set on its side. The shutter assembly has a small rare earth magnet secured to the counterweight. The magnet's position is sensed by two Hall effect detectors that sense the state of the shutter during operation. When an exposure is to be started or ended, the position of the shutter is checked one second after energizing or de-energizing the solenoid, and every second thereafter during irradiation. The message "Shutter Not Open" or "Shutter Stuck Open" is displayed, solenoid power is removed, and the program waits if the shutter status is not sensed to be as expected.

A shutter which will not close by command from the front panel can be closed manually by disassembling the instrument's case and gaining access to the top of the irradiator. (Note that, even with the shutter in open position, the only region actually exposed to beta radiation is the sample which is positioned directly below the source, as long as the source shield assembly remains uncompromised.)

Any condition that requires disassembly or modification of the source shield must be performed in the presence of an ORNL RCT, who shall approve conditions under which the operation will be performed, monitor radiological conditions during the operation, and determine the suitability of the equipment for return to service at the operation's conclusion.

\subsection{Operational Requirements}

Assurance that the equipment is operating normally, that safety systems work as expected, and that data are retrievable and can be associated with the Experimenter who generated it is primarily the responsibility of the PI through Quarterly Checks and Maintenance. However, the individual Experimenter is also responsible for verifying through Operational Checks that equipment is working properly for each experiment and that records of observations and measurements are properly recorded. The Experimenter is also responsible for reporting any abnormal equipment behavior to the PI for resolution.

\subsection{Records Keeping and Data Management}

A TL/OSL daily logbook shall contain a brief description of activities involving the DOSAR thermoluminescence/optically stimulated luminescence analytical equipment and related experiments. These descriptions shall include the date and a description of the activity, and shall be signed by the Experimenter performing the work. The daily logbook shall also contain appropriate entries concerning equipment calibration and maintenance.

Data generated by the TL/OSL analytical systems shall be stored in electronic format on the control computer's hard drive. The subdirectory structure under which these data are stored shall identify the Experimenter generating the data. 


\subsection{Planned Maintenance/Safety Checks/Calibrations}

Facility and equipment maintenance, operational tests of safety systems, and tests or recalibration of calibrated equipment will be performed periodically. Specific actions and their frequencies are identified in Appendix B. Procedures for selected activities related to these actions are provided in Appendix C.

\subsection{Documentation Requirements}

This report provides a readily available source of information about operational procedures and requirements for using TL and OSL instrumentation at DOSAR. It must be reviewed for accuracy by officers of the Life Sciences Division to ensure that it reflects the relevant ORNL policy guidance, and it must be read and understood by individual Experimenters before starting TL or OSL research at DOSAR.

\subsection{Signature Record}

Each TL/OSL Experimenter is required to read and understand the current Protocols, as indicated by signature on the Signature Record (Appendix D).

\subsection{Document Revision History and Approval}

Significant changes to these protocols are initiated by the PI and implemented in a Revision to this report. Revisions are submitted for inclusion in the ORNL Comprehensive Publications and Presentations Registry and must be reviewed and approved by the PI, the LSD Quality Assurance Representative and the LSD Radiation Control Officer, as indicated by their signatures on the Approval Page (Appendix E).

\subsection{Quality Assurance Plan Description}

TL/OSL research at DOSAR conforms to the ORNL Quality Assurance (QA) Program, which supports ORNL's quest for scientific and operational excellence within nuclear facilities, radiological areas, and within programs or activities that may impact nuclear safety; and which also provides a structured, yet non-mandatory, framework for all other activities at the Laboratory. The program is specifically designed to meet the requirements of 10 CFR 830 Subpart A, which is applicable to nuclear facilities, radiological areas, programs and activities that may impact nuclear or radiological safety. The program incorporates appropriate tools from a number of quality standards including the NQA-1 "Quality Assurance Requirements for Nuclear Facility Application", DOE O 414.1, and DOE G 414.1-2 "Quality Assurance Management System Guide for use with 10 CFR 830.120 and DOE O 414.1". Using these consensus standards as tools in the development of this program ensures the delivery of a comprehensive, process-based, integrated Quality Program that meets the U.S. Department of Energy's requirements and programmatic needs. Effective application of the program begins with the Laboratory's Mission and Agenda. 


\title{
Appendix A: Training Outlines for Experimenters
}

\section{GOALS}
a. Laboratory Safety
b. ALARA
c. Environmental Protection

II. RESPONSIBILITIES
a. TL/OSL PI
b. TL/OSL Experimenter
c. Radiation Control Officer/Radiation Safety Services Representative

\section{REQUIREMENTS FOR USING TL/OSL EQUIPMENT}

a. Personnel radiation monitoring

b. Safety training required by ORNL

c. Task-based training for radiation generating devices

d. Familiarity with the operating procedures and equipment

\author{
IV. TYPES OF HAZARDS \\ a. Radiological \\ b. Electrical \\ c. Incidental (Ovens, Vacuum System, Pressurized Gases)
}

\section{RADIATION HAZARDS}

a. Beta sources

1. Description

2. Dose rates and hazards

b. Alpha sources

1. Description

2. Hazards

\section{PROCEDURES}
a. Authorized users
b. General safety
c. Written procedures
d. Housekeeping
e. Mounting samples
g. Preparatory steps
h. Analyzing samples
i. Irradiating samples
j. Shutdown
k. Recordkeeping and logbooks

\section{COMPUTER CONTROL SYSTEM}

a. Using the personal computer control system (1100 TL System, 2200 OSL System)

b. Keypad operation (801E Irradiator)

c. Data generation and storage 


\section{Appendix B: Schedule for Planned Maintenance, Safety Checks, and Calibrations}

1. Operational Checks ( prior to each use)
A. Verify computer operation
B. Verify sample transport
C. Verify irradiator operation

2. Quarterly Checks and Maintenance
A. Verify $801 \mathrm{E}$ Irradiator operation
B. Verify correct operation of 2200 OSL System interlock
C. Test operation of emergency egress lighting
D. Verify operation of lighted 'EXIT' signs
E. Backup computer record
F. Verify record entries
G. Verify that Authorized User List is current
H. Forward copies of newly completed task-based Radiation-Generating Device training to LSD Training Officer

\section{Additional Tests}

A. Perform periodic leak tests of radioactive sealed sources

Sealed radioactive sources in the Daybreak Model $801 \mathrm{E}$ Irradiator $\left({ }^{90} \mathrm{Sr} /{ }^{90} \mathrm{Y}\right.$ and $\left.{ }^{241} \mathrm{Am}\right)$ and the Model 2200 OSL System $\left({ }^{90} \mathrm{Sr} /{ }^{90} \mathrm{Y}\right)$ are leak-tested for integrity periodically by ORNL Radiological Control Technicians as part of ORNL's Radiation Source Control Program. The frequency and procedures for leak-testing radiation sources are consistent with requirements of the Code of Federal Regulations (10CFR835) and ORNL SBMS Program Description: Radiological Protection.

B. Check under Irradiator sample platter for lost samples that may cause the platter rotating mechanism to jam 


\section{Appendix C: Procedures for Routine Maintenance, Safety Checks and Calibrations}

1. Operational Checks

A. Complete preparatory steps as detailed in Sections 4.2.1 (Model 1100 TL) or 4.3.1 (Model 2200 OSL).

B. Verify both system response and irradiator operation (2200 OSL only). This may be done either by using a script file or by manually entering the operational parameters, as detailed in Sections 4.2.2 (Model $1100 \mathrm{TL}$ ) and 4.3.2 (Model 2200 OSL).

C. Verify correct operation of the Model 801E Irradiator using the instructions in Section 4.4.

2. Quarterly Checks and Maintenance

A. Verify Model 801E Irradiator operation and Model 2200 OSL sample irradiator operation as specified in Operational Checks.

B. Verify correct operation of the Model 2200 OSL radiation source shutter interlock by attempting to activate the irradiator from the computer console with the top lid of the 2200 raised. Failure of the source shutter to open indicates that the interlock is working correctly. A portable radiation monitoring device placed in the vicinity of the opening beneath the source shutter should be used to determine shutter status. ${ }^{6}$ NOTE: If the source shutter opens with the Model 2200 OSL lid raised, then abort the operation immediately, secure the 2200 system and notify the PI.

\section{Additional Tests}

A. Daybreak recommends wipe testing the 20-30 sample positions (which fall under the sources in the course of irradiations) of the $801 \mathrm{E}$ Irradiator and the 2200 OSL system using the following procedure:

1. Enter LOAD mode

2. Use the arrow keys to bring each sample position in turn to the load door for wiping with a filter paper moistened with alcohol. Be careful not to touch the white numbers on the sample platter, as they are not impervious to solvents.

3. Evaluate the wipe paper for alpha and beta activity using a standard comparative technique approved by the local authority.

B. Samples that have been lost during loading of the Model 801E Irradiator and that have fallen under the sample platter are retrieved by turning the irradiator on its side and removing the base.

\footnotetext{
${ }^{6} \mathrm{~A}$ dose rate of $\sim 5 \mathrm{mrad} / \mathrm{h}$, measured using an open-window Geiger-Mueller detector directly under the closed source shutter, is typical. Much higher dose rates will result with the shutter open, even when the detector is removed several centimeters from the shutter opening.
} 


\section{Appendix D: Signature Record}

I have read and understand the TL/OSL research protocols described in this manual and agree to follow the guidelines provided herein:

Date $\quad$ Printed Name

Signature

Revision Date 


\section{Appendix E: Approval Page}

Reviewed and Updated

J. S. Bogard

Principal Investigator

Date

Reviewed and Approved

J. S. Bogard

Health Physics \& Radiological Protection Program Leader Date

Reviewed and Approved

B. D. Embleton

LSD Radiation Control Officer

Date

Reviewed and Approved

J. L. Wagner

LSD Quality Assurance Coordinator

Date

Revision Distributed

Principal Investigator

Revision Distributed Principal Investigator

Revision Distributed

Principal Investigator
J. S. Bogard

J. S. Bogard

J. S. Bogard

Date

Reviewed without Revision 


\section{Internal Distribution}

$\begin{array}{llll}\text { 1-7. } & \text { J.S. Bogard } & 10 . & \text { T. Vo-Dinh } \\ 8 . & \text { B. D. Embleton } & 11-12 . & \text { Laboratory Records } \\ 9 . & \text { J. L. Wagner } & 13 . & \text { Laboratory Records - RC }\end{array}$

\section{External Distribution}

14. S. M. Bernal, Department of Nuclear Engineering and Radiological Sciences, University of Michigan, Ann Arbor MI 48109-2104

15. J. M. Elam, Department of Anthropology, University of Tennessee, Knoxville TN 37996-0720

16. A. Kramer, Department of Anthropology, University of Tennessee, Knoxville TN 37996-0720 